\title{
Multiplicity of positive solutions for Sturm-Liouville boundary value problems of fractional differential equations with $p$-Laplacian
}

Hongling Lu, Zhenlai Han ${ }^{*}$ and Shurong Sun

\author{
"Correspondence: \\ hanzhenlai@163.com \\ School of Mathematical Sciences, \\ University of Jinan, Jinan, Shandong \\ 250022, P.R. China
}

\begin{abstract}
In this article, we investigate the Sturm-Liouville boundary value problems of fractional differential equations with $p$-Laplacian

$$
\left\{\begin{array}{l}
D_{0^{+}}^{\beta}\left(\phi_{p}\left(D_{0^{+}}^{\alpha} u(t)\right)\right)+f(t, u(t))=0, \quad 0<t<1, \\
\xi u(0)-\eta u^{\prime}(0)=0, \quad \gamma u(1)+\delta u^{\prime}(1)=0, \quad D_{0^{+}}^{\alpha} u(0)=0,
\end{array}\right.
$$

where $1<\alpha \leq 2,0<\beta \leq 1, D_{0^{+}}^{\alpha}, D_{0^{+}}^{\beta}$ are the standard Caputo fractional derivatives, $\phi_{p}(s)=|s|^{p-2} s, p>1, \phi_{p}^{-1}=\phi_{q}, 1 / p+1 / q=1, \xi, \eta, \gamma, \delta \geq 0, \rho:=\xi \gamma+\xi \delta+\eta \gamma>0$, and $f:[0,1] \times[0,+\infty) \rightarrow[0,+\infty)$ is continuous. By means of the properties of the Green's function, Leggett-Williams fixed-point theorems, and fixed-point index theory, several new sufficient conditions for the existence of at least two or at least three positive solutions are obtained. As an application, an example is given to demonstrate the main result.
\end{abstract}

MSC: 34A08; 34B18; 35J05

Keywords: Sturm-Liouville boundary value problem; positive solution of fractional differential equation; Leggett-Williams fixed-point theorem; fixed-point index theory; p-Laplacian operator

\section{Introduction}

During the past decades, much attention has been focused on the study of equations with $p$-Laplacian differential operator. The motivation for those works stems from the applications in the modeling of different physical and natural phenomena: non-Newtonian mechanics [1], system of Monge-Kantorovich partial differential equations [2], population biology [3], nonlinear flow laws [4], combustion theory [5]. There exist a very large number of papers devoted to the existence of solutions for the equation with $p$-Laplacian operator.

The ordinary differential equation with $p$-Laplacian operator

$$
\left(\phi_{p}\left(u^{\prime}(t)\right)\right)^{\prime}+f(t, u(t))=0, \quad 0<t<1
$$

subject to various boundary conditions, has been studied by many authors, see [6, 7] and the references therein.

@2014 Lu et al.; licensee Springer. This is an Open Access article distributed under the terms of the Creative Commons Attribution License (http://creativecommons.org/licenses/by/2.0), which permits unrestricted use, distribution, and reproduction in any medium, provided the original work is properly cited. 
The existence of positive solutions of the differential equation with $p$-Laplacian operator

$$
\left(\phi_{p}\left(u^{\prime}(t)\right)\right)^{\prime}+q(t) f\left(t, u(t), u^{\prime}(t)\right)=0, \quad 0<t<1
$$

satisfying different boundary conditions have been established by using fixed-point theorems and monotone iterative technique, see $[8,9]$ and the references therein.

In [10], Hai considered the existence of positive solutions for the boundary value problem

$$
\left\{\begin{array}{l}
\left(q(t) \phi\left(u^{\prime}\right)\right)^{\prime}+\lambda f(t, u(t))=0, \quad r<t<R, \\
a u(r)-b \phi^{-1}(q(r)) u^{\prime}(r)=0, \quad c u(R)+d \phi^{-1}(q(R)) u^{\prime}(R)=0,
\end{array}\right.
$$

where $\phi\left(u^{\prime}\right)=\left|u^{\prime}\right|^{p-2} u^{\prime}, p>1, f:(r, R) \times(0, \infty) \rightarrow \mathbb{R}$ and $\lambda$ is a positive parameter, $f$ is $p$-superlinear or $p$-sublinear at $\infty$ and maybe singular at $u=0$.

However, few papers can be found in the literature on the existence of multiple positive solutions for the third-order Sturm-Liouville boundary value problem with $p$-Laplacian.

In [11], Zhai and Guo studied the third-order Sturm-Liouville boundary value problem with $p$-Laplacian

$$
\left\{\begin{array}{l}
\left(\phi_{p}\left(u^{\prime \prime}(t)\right)\right)^{\prime}+f(t, u(t))=0, \quad 0<t<1, \\
\alpha u(0)-\beta u^{\prime}(0)=0, \quad \gamma u(1)+\delta u^{\prime}(1)=0, \quad u^{\prime \prime}(0)=0
\end{array}\right.
$$

where $\phi_{p}(s)=|s|^{p-2} s, p>1,\left(\phi_{p}\right)^{-1}=\phi_{q}, 1 / p+1 / q=1, \alpha, \beta, \gamma, \delta \geq 0, f \in C([0,1] \times$ $[0, \infty),[0, \infty))$. By means of the Leggett-Williams fixed-point theorems, some existence and multiplicity results of positive solutions are obtained. In later work, Yang and Yan [12] also studied the above problem by means of the fixed-point index method.

Recently, fractional differential equations have been of great interest. The motivation for those works stems from both the intensive development of the theory of fractional calculus itself and the applications such as economics, engineering and other fields [13-17]. Much attention has been focused on the study of the existence and multiplicity of solutions or positive solutions for boundary value problems of fractional differential equations by the use of techniques of nonlinear analysis (fixed-point theorems [18-25], upper and lower solutions method [26], fixed-point index theory [27, 28], coincidence theory [29], etc.).

Although the boundary value problems of fractional differential equation with $p$ Laplacian have been studied in many literature, only few papers can be found in the literature on the existence of multiple positive solutions for the Sturm-Liouville boundary value problems of fractional differential equations with $p$-Laplacian. As the extension and supplement of some results in $[11,12]$, in this article, we investigate the Sturm-Liouville boundary value problems of fractional differential equations with $p$-Laplacian subject Robin boundary value conditions

$$
\left\{\begin{array}{l}
D_{0^{+}}^{\beta}\left(\phi_{p}\left(D_{0^{+}}^{\alpha} u(t)\right)\right)+f(t, u(t))=0, \quad 0<t<1, \\
\xi u(0)-\eta u^{\prime}(0)=0, \quad \gamma u(1)+\delta u^{\prime}(1)=0, \quad D_{0^{+}}^{\alpha} u(0)=0,
\end{array}\right.
$$

where $1<\alpha \leq 2,0<\beta \leq 1, D_{0^{+}}^{\alpha}, D_{0^{+}}^{\beta}$ are the standard Caputo fractional derivatives, $\phi_{p}(s)=|s|^{p-2} s, p>1, \phi_{p}^{-1}=\phi_{q}, 1 / p+1 / q=1, \xi, \eta, \gamma, \delta \geq 0, \rho:=\xi \gamma+\xi \delta+\eta \gamma>0$, and 
$f:[0,1] \times[0,+\infty) \rightarrow[0,+\infty)$ is continuous. By means of the properties of the Green's function, Leggett-Williams fixed-point theorems and fixed-point index theory, we establish the existence of at least two or at least three positive solutions for the Sturm-Liouville boundary value problem (1.1). As an application, an example is given to demonstrate the main result.

The rest of this paper is organized as follows. In Section 2, we shall introduce some definitions and lemmas to prove our main results. In Section 3, we state our main results. We prove our main results by Leggett-Williams fixed-point theorems and fixed-point index theory in Section 4. As an application, an example is presented to illustrate our main result in Section 5.

\section{Preliminaries and lemmas}

For the convenience of the reader, we give some background materials from fractional calculus theory to facilitate analysis of problem (1.1). These materials can be found in the recent literature, see [14, 17, 18, 31-33].

Definition 2.1 ([17]) The Riemann-Liouville fractional integral of order $\alpha>0$ of a function $y:(0,+\infty) \rightarrow \mathbb{R}$ is given by

$$
I_{0^{+}}^{\alpha} y(t)=\frac{1}{\Gamma(\alpha)} \int_{0}^{t}(t-s)^{\alpha-1} y(s) d s
$$

provided the right side is pointwise defined on $(0,+\infty)$.

Definition 2.2 ([17]) The Caputo fractional derivative of order $\alpha>0$ of a continuous function $y:(0,+\infty) \rightarrow \mathbb{R}$ is given by

$$
D_{0^{+}}^{\alpha} y(t)=\frac{1}{\Gamma(n-\alpha)} \int_{0}^{t} \frac{y^{(n)}(s)}{(t-s)^{\alpha-n+1}} d s
$$

where $n$ is the smallest integer greater than or equal to $\alpha$, provided that the right side is pointwise defined on $(0,+\infty)$.

Remark 2.1 ([14]) By Definition 2.2, under natural conditions on the function $f(t)$, as $\alpha \rightarrow n$ the Caputo derivative becomes a conventional $n$th derivative of the function $f(t)$.

Remark 2.2 ([18]) As a basic example, we have

$$
D_{0^{+}}^{\alpha} t^{\mu}=\mu(\mu-1) \cdots(\mu-n+1) \frac{\Gamma(1+\mu-n)}{\Gamma(1+\mu-\alpha)} t^{\mu-\alpha}, \quad \text { for } t \in(0,+\infty),
$$

given in particular that $D_{0^{+}}^{\alpha} t^{\mu}=0, \mu=0,1, \ldots, n-1$, where $D_{0^{+}}^{\alpha}$ is the Caputo fractional derivative, and $n$ is the smallest integer greater than or equal to $\alpha$.

From the definition of the Caputo derivative and Remark 2.2, we can obtain the following statement.

Lemma 2.1 ([17]) Let $\alpha>0$. Then the fractional differential equation

$$
D_{0^{+}}^{\alpha} u(t)=0
$$


has

$$
u(t)=c_{0}+c_{1} t+c_{2} t^{2}+\cdots+c_{n-1} t^{n-1}, \quad c_{i} \in \mathbb{R}, i=0,1,2, \ldots, n-1
$$

as the unique solution, where $n$ is the smallest integer greater than or equal to $\alpha$.

Lemma 2.2 ([17]) Let $\alpha>0$. Assume that $u, D_{0^{+}}^{\alpha} u \in L(0,1)$. Then the following equality holds:

$$
I_{0^{+}}^{\alpha} D_{0^{+}}^{\alpha} u(t)=u(t)+c_{0}+c_{1} t+c_{2} t^{2}+\cdots+c_{n-1} t^{n-1},
$$

for some $c_{i} \in \mathbb{R}, i=0,1,2, \ldots, n-1$, where $n$ is the smallest integer greater than or equal to $\alpha$.

Lemma 2.3 Let $y \in C[0,1]$ and $1<\alpha \leq 2$. Then the boundary value problem of the fractional differential equation

$$
\left\{\begin{array}{l}
D_{0^{+}}^{\alpha} u(t)+y(t)=0, \quad 0<t<1, \\
\xi u(0)-\eta u^{\prime}(0)=0, \quad \gamma u(1)+\delta u^{\prime}(1)=0
\end{array}\right.
$$

has a unique solution,

$$
u(t)=\int_{0}^{1} G(t, s) y(s) d s
$$

where

$$
G(t, s)= \begin{cases}-\frac{(t-s)^{\alpha-1}}{\Gamma(\alpha)}+\frac{\eta+\xi t}{\rho}\left[\frac{\gamma}{\Gamma(\alpha)}(1-s)^{\alpha-1}+\frac{\delta}{\Gamma(\alpha-1)}(1-s)^{\alpha-2}\right], & s \leq t \\ \frac{\eta+\xi t}{\rho}\left[\frac{\gamma}{\Gamma(\alpha)}(1-s)^{\alpha-1}+\frac{\delta}{\Gamma(\alpha-1)}(1-s)^{\alpha-2}\right], & t \leq s .\end{cases}
$$

Proof By the Lemma 2.2, we can reduce the equation of problem (2.1) to an equivalent integral equation

$$
u(t)=-I_{0^{+}}^{\alpha} y(t)-c_{0}-c_{1} t=-\int_{0}^{t} \frac{(t-s)^{\alpha-1}}{\Gamma(\alpha)} y(s) d s-c_{0}-c_{1} t,
$$

for some constants $c_{0}, c_{1} \in \mathbb{R}$. Moreover, we have

$$
u^{\prime}(t)=-\frac{1}{\Gamma(\alpha-1)} \int_{0}^{t}(t-s)^{\alpha-2} y(s) d s-c_{1} .
$$

From the boundary conditions $\xi u(0)-\eta u^{\prime}(0)=0, \gamma u(1)+\delta u^{\prime}(1)=0$, we have

$$
\xi c_{0}=\eta c_{1}, \quad \gamma\left(-I_{0^{+}}^{\alpha} y(1)-c_{0}-c_{1}\right)+\delta\left(-I_{0^{+}}^{\alpha-1} y(1)-c_{1}\right)=0 .
$$

So,

$$
c_{0}=-\frac{\eta}{\rho}\left(\gamma I_{0^{+}}^{\alpha} y(1)+\delta I_{0^{+}}^{\alpha-1} y(1)\right), \quad c_{1}=-\frac{\xi}{\rho}\left(\gamma I_{0^{+}}^{\alpha} y(1)+\delta I_{0^{+}}^{\alpha-1} y(1)\right) .
$$


Hence, the unique solution of (2.1) is

$$
\begin{aligned}
u(t)= & -\int_{0}^{t} \frac{(t-s)^{\alpha-1}}{\Gamma(\alpha)} y(s) d s+\frac{\eta}{\rho}\left(\gamma \int_{0}^{1} \frac{(1-s)^{\alpha-1}}{\Gamma(\alpha)} y(s) d s+\delta \int_{0}^{1} \frac{(1-s)^{\alpha-2}}{\Gamma(\alpha-1)} y(s) d s\right) \\
& +\frac{\xi t}{\rho}\left(\gamma \int_{0}^{1} \frac{(1-s)^{\alpha-1}}{\Gamma(\alpha)} y(s) d s+\delta \int_{0}^{1} \frac{(1-s)^{\alpha-2}}{\Gamma(\alpha-1)} y(s) d s\right) \\
= & \int_{0}^{t}\left(-\frac{(t-s)^{\alpha-1}}{\Gamma(\alpha)}+\frac{\eta \gamma+\xi \gamma t}{\rho \Gamma(\alpha)}(1-s)^{\alpha-1}+\frac{\eta \delta+\xi \delta t}{\rho \Gamma(\alpha-1)}(1-s)^{\alpha-2}\right) y(s) d s \\
& +\int_{t}^{1}\left(\frac{\eta \gamma+\xi \gamma t}{\rho \Gamma(\alpha)}(1-s)^{\alpha-1}+\frac{\eta \delta+\xi \delta t}{\rho \Gamma(\alpha-1)}(1-s)^{\alpha-2}\right) y(s) d s \\
= & \int_{0}^{1} G(t, s) y(s) d s,
\end{aligned}
$$

which completes the proof.

Lemma 2.4 Let $1<\alpha \leq 2,0<\beta \leq 1$. Then the boundary value problem of the fractional differential equation

$$
\left\{\begin{array}{l}
D_{0^{+}}^{\beta}\left(\phi_{p}\left(D_{0^{+}}^{\alpha} u(t)\right)\right)+f(t, u(t))=0, \quad 0<t<1, \\
\xi u(0)-\eta u^{\prime}(0)=0, \quad \gamma u(1)+\delta u^{\prime}(1)=0, \quad D_{0^{+}}^{\alpha} u(0)=0
\end{array}\right.
$$

has a unique solution,

$$
u(t)=\int_{0}^{1} G(t, s) \phi_{q}\left(\int_{0}^{s} \frac{(s-\tau)^{\beta-1}}{\Gamma(\beta)} f(\tau, u(\tau)) d \tau\right) d s
$$

where $G(t, s)$ is defined as (2.2).

Proof From Lemma 2.2 and the boundary value problem (2.3), we have

$$
\begin{aligned}
I_{0^{+}}^{\beta} D_{0^{+}}^{\beta}\left(\phi_{p}\left(D_{0^{+}}^{\alpha} u(t)\right)\right) & =-I_{0^{+}}^{\beta} f(t, u(t)) \\
& =\phi_{p}\left(D_{0^{+}}^{\alpha} u(t)\right)+c_{0}, \quad \text { for } c_{0} \in \mathbb{R},
\end{aligned}
$$

that is

$$
\phi_{p}\left(D_{0^{+}}^{\alpha} u(t)\right)=-\int_{0}^{t} \frac{(t-\tau)^{\beta-1}}{\Gamma(\beta)} f(\tau, u(\tau)) d \tau-c_{0}, \quad \text { for } c_{0} \in \mathbb{R} .
$$

By $D_{0^{+}}^{\alpha} u(0)=0$, we have $c_{0}=0$. So, $D_{0^{+}}^{\alpha} u(t)+\phi_{q}\left(\int_{0}^{t} \frac{(t-\tau)^{\beta-1}}{\Gamma(\beta)} f(\tau, u(\tau)) d \tau\right)=0$. Thus, the boundary value problem (2.3) is equivalent to the following problem:

$$
\begin{aligned}
& D_{0^{+}}^{\alpha} u(t)+\phi_{q}\left(\int_{0}^{t} \frac{(t-\tau)^{\beta-1}}{\Gamma(\beta)} f(\tau, u(\tau)) d \tau\right)=0, \quad 0<t<1, \\
& \xi u(0)-\eta u^{\prime}(0)=0, \quad \gamma u(1)+\delta u^{\prime}(1)=0 .
\end{aligned}
$$


Lemma 2.3 implies that boundary value problem (2.3) has a unique solution,

$$
u(t)=\int_{0}^{1} G(t, s) \phi_{q}\left(\int_{0}^{s} \frac{(s-\tau)^{\beta-1}}{\Gamma(\beta)} f(\tau, u(\tau)) d \tau\right) d s
$$

which completes the proof.

Lemma 2.5 The Green's function $G(t, s)$ defined by $(2.2)$ is continuous on $[0,1] \times[0,1]$. Assume $\eta>\frac{2-\alpha}{\alpha-1} \xi$, then $G(t, s)$ also has the following properties:

(1) $G(t, s)>0$, for $t, s \in[0,1]$;

(2) $G(t, s) \leq G(s, s)$, for $t, s \in(0,1)$;

(3) there exists a positive number $\lambda$ such that $G(t, s) \geq \lambda G(s, s)$, for $t, s \in[0,1]$, where $\lambda=\min \left\{\frac{4 \xi \gamma \delta((\alpha-2) \xi+(\alpha-1) \eta)}{((\alpha-1) \xi \delta+\xi \gamma-\eta \gamma)+4 \xi \gamma((\alpha-1) \eta \delta+\eta \gamma)}, \frac{4 \xi \eta \gamma \delta((\alpha-2) \xi+(\alpha-1) \eta)}{((\alpha-1) \xi \delta+\xi \gamma-\eta \gamma)+4 \xi \gamma((\alpha-1) \eta \delta+\eta \gamma)}\right\}<1$.

The method of proof is similar to Lemma 3.2 in [30], and we omit it here.

Definition 2.3 ([31]) Let $E$ be a real Banach space and $P$ be a nonempty, convex closed set in $E$. We say that $P$ is a cone if it satisfies the following properties:

(i) $\lambda u \in P$ for $u \in P, \lambda \geq 0$;

(ii) $u,-u \in P$ implies $u=\theta$, where $\theta$ denotes the null element of $E$.

If $P \subset E$ is a cone, we denote the order induced by $P$ on $E$ by $\leq$. For $u, v \in P$, we write $u \leq v$ if $v-u \in P$.

Definition 2.4 ([31]) The map $\varphi$ is said to be a nonnegative continuous concave functional on $P$ of a real Banach space $E$ provided that $\varphi: P \rightarrow[0, \infty)$ is continuous and

$$
\varphi(t x+(1-t) y) \geq t \varphi(x)+(1-t) \varphi(y)
$$

for all $x, y \in P$ and $0 \leq t \leq 1$.

Definition 2.5 ([31]) Let $0<a<b$ be given and let $\varphi$ be a nonnegative continuous concave functional on the cone $P$. Define the convex sets $P_{r}, \bar{P}_{r}$ and $P(\varphi, a, b)$ by $P_{r}=\{y \in P\|\| y \|<r\}$, $\bar{P}_{r}=\{y \in P \mid\|y\| \leq r\}, P(\varphi, a, b)=\{y \in P \mid a \leq \varphi(y),\|y\| \leq b\}$.

Lemma 2.6 (Leggett-Williams [32]) Let $T: \bar{P}_{c} \rightarrow \bar{P}_{c}$ be a completely continuous operator and let $\varphi$ be a nonnegative continuous concave functional on $P$ such that $\varphi(y) \leq\|y\|$ for all $y \in \bar{P}_{c}$. Suppose that there exist $0<a<b<d \leq c$ such that

$\left(\mathrm{A}_{1}\right) \quad\{y \in P(\varphi, b, d) \mid \varphi(y)>b\} \neq \emptyset$ and $\varphi(T y)>b$ for $y \in P(\varphi, b, d)$;

$\left(\mathrm{A}_{2}\right)\|T y\|<a$ for $\|y\| \leq a$;

$\left(\mathrm{A}_{3}\right) \varphi(T y)>b$ for $y \in P(\varphi, b, c)$ with $\|T y\|>d$.

Then $T$ has at least three fixed points $y_{1}, y_{2}$, and $y_{3}$ in $\bar{P}_{c}$ satisfying $\left\|y_{1}\right\|<a, \varphi\left(y_{2}\right)>b$, $\left\|y_{3}\right\|>a$, and $\varphi\left(y_{3}\right)<b$.

Lemma 2.7 ([32]) Let $T: \bar{P}_{c} \rightarrow P$ be a completely continuous operator and let $\varphi$ be a nonnegative continuous concave functional on $P$ such that $\varphi(y) \leq\|y\|$ for all $y \in \bar{P}_{c}$. Suppose that there exist $0<a<b<c$ such that 
(B) $\{y \in P(\varphi, b, c) \mid \varphi(y)>b\} \neq \emptyset$, and $\varphi($ Ty $)>b$ for $y \in P(\varphi, b, c)$;

$\left(\mathrm{B}_{2}\right)\|T y\|<a$ for $\|y\| \leq a$;

(B) $\varphi(T y)>\frac{b}{c}\|T y\|$ for $y \in \bar{P}_{c}$ with $\|T y\|>c$.

Then $T$ has at least two fixed points $y_{1}$ and $y_{2}$ in $\bar{P}_{c}$ satisfying $\left\|y_{1}\right\|<a,\left\|y_{2}\right\|>a$ and $\varphi\left(y_{2}\right)<b$.

Lemma 2.8 ([31]) Let $P$ be a closed convex set in a Banach space $E$ and let $\Omega$ be a bounded open set such that $\Omega_{p}:=\Omega \cap P \neq \emptyset$. Let $T: \bar{\Omega}_{p} \rightarrow P$ be a compact map. Suppose that $x \neq T x$ for all $x \in \partial \Omega_{p}$.

$\left(C_{1}\right)$ (Existence) If $i\left(T, \Omega_{p}, P\right) \neq 0$, then $T$ has a fixed point in $\Omega_{p}$.

$\left(C_{2}\right)$ (Normalization) If $u \in \Omega_{p}$, then $i\left(\hat{u}, \Omega_{p}, P\right)=1$, where $\hat{u}(x)=u$ for $x \in \bar{\Omega}_{p}$.

$\left(C_{3}\right)$ (Homotopy) Let $v:[0,1] \times \bar{\Omega}_{p} \rightarrow P$ be a compact map such that $x \neq v(t, x)$ for $x \in \partial \Omega_{p}$ and $t \in[0,1]$. Then $i\left(v(0, \cdot), \Omega_{p}, P\right)=i\left(v(1, \cdot), \Omega_{p}, P\right)$.

$\left(C_{4}\right)$ (Additivity) If $U_{1}, U_{2}$ are disjoint relatively open subsets of $\Omega_{p}$ such that $x \neq T x$ for $x \in \bar{\Omega}_{p} \backslash\left(U_{1} \cup U_{2}\right)$, then $i\left(T, \Omega_{p}, P\right)=i\left(T, U_{1}, P\right)+i\left(T, U_{2}, P\right)$, where $i\left(T, U_{j}, P\right)=$ $i\left(\left.T\right|_{\bar{u}_{j}}, U_{j}, P\right)(j=1,2)$.

Lemma 2.9 ([33]) Let $P$ be a cone in a Banach space E. For $q>0$, define $\Omega_{q}=\{x \in p \mid\|x\|<$ q\}. Assume that $T: \bar{\Omega}_{q} \rightarrow P$ is a compact map such that $x \neq$ Tx for $x \in \partial \Omega_{q}$. Thus, one has the following conclusions:

$\left(\mathrm{D}_{1}\right)$ if $\|x\| \leq\|T x\|$ for $x \in \partial \Omega_{q}$, then $i\left(T, \Omega_{q}, P\right)=0$;

$\left(\mathrm{D}_{2}\right)$ if $\|x\| \geq\|T x\|$ for $x \in \partial \Omega_{q}$, then $i\left(T, \Omega_{q}, P\right)=1$.

\section{Main theorems}

In this section, let $E=C[0,1]$ be the Banach space of continuous functions endowed with $\|u\|=\max _{0 \leq t \leq 1}|u(t)|$, and the ordering $x \leq y$ if $x(t) \leq y(t)$ for all $t \in[0,1]$. Define the cone $P \subset E$ by

$$
P=\left\{u \in E \mid u(t) \geq 0, \min _{1 / 4 \leq t \leq 3 / 4} u(t) \geq \lambda\|u\|\right\}
$$

where $\lambda$ is given as in Lemma 2.5 .

For convenience of the reader, we denote

$$
\begin{aligned}
& M=\phi_{q}(\Gamma(\beta+1))\left(\int_{0}^{1} G(s, s) d s\right)^{-1}, \quad N=\frac{2}{\lambda} \phi_{q}\left(4^{\beta} \Gamma(\beta+1)\right)\left(\int_{\frac{1}{4}}^{\frac{3}{4}} G\left(\frac{1}{2}, s\right) d s\right)^{-1}, \\
& f_{0}:=\lim _{u \rightarrow 0^{+}} \min _{0 \leq t \leq 1} \frac{f(t, u)}{\phi_{p}(u)}, \quad f_{\infty}:=\lim _{u \rightarrow+\infty} \max _{0 \leq t \leq 1} \frac{f(t, u)}{\phi_{p}(u)} .
\end{aligned}
$$

Lemma 3.1 Let $T: P \rightarrow$ E be the operator defined by

$$
T u(t):=\int_{0}^{1} G(t, s) \phi_{q}\left(\int_{0}^{s} \frac{(s-\tau)^{\beta-1}}{\Gamma(\beta)} f(\tau, u(\tau)) d \tau\right) d s .
$$

Then $T: P \rightarrow P$ is completely continuous. 
Proof By Lemma 2.5, we have

$$
\begin{aligned}
&\|T u(t)\| \leq \int_{0}^{1} G(s, s) \phi_{q}\left(\int_{0}^{s} \frac{(s-\tau)^{\beta-1}}{\Gamma(\beta)} f(\tau, u(\tau)) d \tau\right) d s \\
& \min _{1 / 4 \leq t \leq 3 / 4}(T u)(t)=\min _{1 / 4 \leq t \leq 3 / 4} \int_{0}^{1} G(t, s) \phi_{q}\left(\int_{0}^{s} \frac{(s-\tau)^{\beta-1}}{\Gamma(\beta)} f(\tau, u(\tau)) d \tau\right) d s \\
& \geq \lambda \int_{0}^{1} G(s, s) \phi_{q}\left(\int_{0}^{s} \frac{(s-\tau)^{\beta-1}}{\Gamma(\beta)} f(\tau, u(\tau)) d \tau\right) d s \\
& \geq \lambda\|T u\| .
\end{aligned}
$$

Thus, $T(P) \subset P$. In view of non-negativity and continuity of $G(t, s), \frac{(s-\tau)^{\beta-1}}{\Gamma(\beta)}$ and $f(t, u(t))$, we find that $T: P \rightarrow P$ is continuous.

Let $\Omega \subset P$ be bounded, i.e., there exists a positive constant $M_{0}$ such that $\|u\| \leq M_{0}$, for all $u \in \Omega$. Let $L=\max _{0 \leq t \leq 1,0 \leq u \leq M_{0}}|f(t, u)|+1$, then, for $u \in \Omega$, we have

$$
\begin{aligned}
|T u(t)| & =\left|\int_{0}^{1} G(t, s) \phi_{q}\left(\int_{0}^{s} \frac{(s-\tau)^{\beta-1}}{\Gamma(\beta)} f(\tau, u(\tau)) d \tau\right) d s\right| \\
& \leq \int_{0}^{1} G(s, s) \phi_{q}\left(\int_{0}^{s} \frac{(s-\tau)^{\beta-1}}{\Gamma(\beta)} f(\tau, u(\tau)) d \tau\right) d s \\
& \leq \int_{0}^{1} G(s, s) \phi_{q}\left(\int_{0}^{s} L \frac{(s-\tau)^{\beta-1}}{\Gamma(\beta)} d \tau\right) d s \\
& =\phi_{q}\left(\frac{L}{\Gamma(\beta+1)}\right) \int_{0}^{1} G(s, s) \phi_{q}\left(s^{\beta}\right) d s .
\end{aligned}
$$

Hence, $T(\Omega)$ is uniformly bounded. Further for any $u \in \Omega$ and $t \in[0,1]$, we have

$$
\begin{aligned}
\left|(T u)^{\prime}(t)\right| \leq & \mid \int_{0}^{t}\left(\frac{-(\alpha-1)(t-s)^{\alpha-2}}{\Gamma(\alpha)}+\frac{\xi \gamma}{\rho \Gamma(\alpha)}(1-s)^{\alpha-1}+\frac{\xi \delta}{\rho \Gamma(\alpha-1)}(1-s)^{\alpha-2}\right) \phi_{q} \\
& \times\left(\int_{0}^{s} L \frac{(s-\tau)^{\beta-1}}{\Gamma(\beta)} d \tau\right) d s \\
& +\int_{t}^{1}\left(\frac{\xi \gamma}{\rho \Gamma(\alpha)}(1-s)^{\alpha-1}+\frac{\xi \delta}{\rho \Gamma(\alpha-1)}(1-s)^{\alpha-2}\right) \phi_{q}\left(\int_{0}^{s} L \frac{(s-\tau)^{\beta-1}}{\Gamma(\beta)} d \tau\right) d s \mid \\
\leq & \frac{\xi}{\rho} \int_{0}^{1}\left(\frac{\gamma}{\Gamma(\alpha)}(1-s)^{\alpha-1}+\frac{\delta}{\Gamma(\alpha-1)}(1-s)^{\alpha-2}\right) \phi_{q}\left(\int_{0}^{s} L \frac{(s-\tau)^{\beta-1}}{\Gamma(\beta)} d \tau\right) d s \\
& +\int_{0}^{t} \frac{(\alpha-1)(t-s)^{\alpha-2}}{\Gamma(\alpha)} \phi_{q}\left(\int_{0}^{s} \frac{(s-\tau)^{\beta-1}}{\Gamma(\beta)} d \tau\right) d s \\
\leq & \frac{\xi}{\rho} \int_{0}^{1}\left(\frac{\gamma}{\Gamma(\alpha)}(1-s)^{\alpha-1}+\frac{\delta}{\Gamma(\alpha-1)}(1-s)^{\alpha-2}\right) \phi_{q}\left(\frac{L s^{\beta}}{\Gamma(\beta+1)}\right) d s \\
& +\int_{0}^{t} \frac{(\alpha-1)(t-s)^{\alpha-2}}{\Gamma(\alpha)} \phi_{q}\left(\frac{L s^{\beta}}{\Gamma(\beta+1)}\right) d s \\
\leq & \phi_{q}\left(\frac{L}{\Gamma(\beta+1)}\right) \frac{\xi \gamma+\alpha \xi \delta+\alpha \rho}{\rho \Gamma(\alpha+1)} .
\end{aligned}
$$


Hence, $\left\|(T u)^{\prime}\right\| \leq \phi_{q}\left(\frac{L}{\Gamma(\beta+1)}\right) \frac{\xi \gamma+\alpha \xi \delta+\alpha \rho}{\rho \Gamma(\alpha+1)}$. For any $0 \leq t_{1} \leq t_{2} \leq 1$ and $u \in \Omega$, we have

$$
\begin{aligned}
\left|\operatorname{Tu}\left(t_{1}\right)-\operatorname{Tu}\left(t_{2}\right)\right| & =\left|\int_{t_{1}}^{t_{2}}(T u)^{\prime}(t) d t\right| \\
& \leq \int_{t_{1}}^{t_{2}}\left|(T u)^{\prime}(t)\right| d t \leq \phi_{q}\left(\frac{L}{\Gamma(\beta+1)}\right) \frac{\xi \gamma+\alpha \xi \delta+\alpha \rho}{\rho \Gamma(\alpha+1)}\left|t_{2}-t_{1}\right| .
\end{aligned}
$$

That is to say, $T(\Omega)$ is equicontinuous. By the Arzela-Ascoli theorem, we see that $T: P \rightarrow P$ is completely continuous. The proof is completed.

We are now ready to prove our main results.

Theorem 3.1 Let $f(t, u)$ be nonnegative continuous on $[0,1] \times[0,+\infty)$. Assume that there exist constants $a, b$ with $b>a>0$ such that

$\left(\mathrm{H}_{1}\right) f(t, u) \geq \phi_{p}(N b)$, for $(t, u) \in\left[\frac{1}{4}, \frac{3}{4}\right] \times\left[b, \frac{b}{\lambda^{2}}\right]$;

$\left(\mathrm{H}_{2}\right) f(t, u)<\phi_{p}(M a)$, for $(t, u) \in[0,1] \times[0, a]$.

Then the boundary value problem (1.1) has at least two positive solutions $u_{1}$ and $u_{2}$ satisfying $\left\|u_{1}\right\|<a, \min _{\frac{1}{4} \leq t \leq \frac{3}{4}} u_{2}(t)<b$ and $\left\|u_{2}\right\|>a$, where $\lambda$ is given as in Lemma 2.5 .

Proof Let $\theta: P \rightarrow[0,+\infty)$ be the nonnegative continuous concave functional defined by

$$
\theta(u)=\min _{\frac{1}{4} \leq t \leq \frac{3}{4}} u(t), \quad u \in P .
$$

Evidently, for each $u \in P$, we have $\theta(u) \leq\|u\|$.

It's easy to see that $T: \bar{P}_{\frac{b}{\lambda^{2}}} \rightarrow P$ is completely continuous and $\frac{b}{\lambda^{2}}>b>a>0$. We choose $u(t)=\frac{b}{\lambda^{2}}$, then

$$
u \in P\left(\theta, b, \frac{b}{\lambda^{2}}\right), \quad \theta(u)=\frac{b}{\lambda^{2}}>b .
$$

So $\left\{u \in P\left(\theta, b, \frac{b}{\lambda^{2}}\right) \mid \theta(u)>b\right\} \neq \emptyset$. Hence, if $u \in P\left(\theta, b, \frac{b}{\lambda^{2}}\right)$, then $b \leq u(t) \leq \frac{b}{\lambda^{2}}$ for $t \in\left[\frac{1}{4}, \frac{3}{4}\right]$. Thus for $t \in\left[\frac{1}{4}, \frac{3}{4}\right]$, from assumption $\left(\mathrm{H}_{1}\right)$, we have

$$
\begin{aligned}
T u\left(\frac{1}{2}\right) & =\int_{0}^{1} G\left(\frac{1}{2}, s\right) \phi_{q}\left(\int_{0}^{s} \frac{(s-\tau)^{\beta-1}}{\Gamma(\beta)} f(\tau, u(\tau)) d \tau\right) d s \\
& \geq \int_{\frac{1}{4}}^{\frac{3}{4}} G\left(\frac{1}{2}, s\right) \phi_{q}\left(\int_{0}^{s} \frac{(s-\tau)^{\beta-1}}{\Gamma(\beta)} f(\tau, u(\tau)) d \tau\right) d s \\
& \geq \int_{\frac{1}{4}}^{\frac{3}{4}} G\left(\frac{1}{2}, s\right) N b \phi_{q}\left(\int_{0}^{s} \frac{(s-\tau)^{\beta-1}}{\Gamma(\beta)} d \tau\right) d s \\
& \geq N b \phi_{q}\left(\frac{1}{4^{\beta} \Gamma(\beta+1)}\right) \int_{\frac{1}{4}}^{\frac{3}{4}} G\left(\frac{1}{2}, s\right) d s=\frac{2 b}{\lambda}>\frac{b}{\lambda} .
\end{aligned}
$$

Consequently,

$$
\min _{\frac{1}{4} \leq t \leq \frac{3}{4}} \operatorname{Tu}(t) \geq \lambda\|T u\|>\lambda \times \frac{b}{\lambda}=b \quad \text { for } \frac{1}{4} \leq t \leq \frac{3}{4}, b \leq u(t) \leq \frac{b}{\lambda^{2}} .
$$


That is,

$$
\theta(T u)>b, \quad \text { for all } u \in P\left(\theta, b, \frac{b}{\lambda^{2}}\right) .
$$

Therefore, condition $\left(\mathrm{B}_{1}\right)$ of Lemma 2.7 is satisfied. Now if $u \in \bar{P}_{a}$, then $\|u\| \leq a$. By assumption $\left(\mathrm{H}_{2}\right)$, we have

$$
\begin{aligned}
\|T u\| & =\max _{0 \leq t \leq 1}|T u(t)|=\max _{0 \leq t \leq 1} \int_{0}^{1} G(t, s) \phi_{q}\left(\int_{0}^{s} \frac{(s-\tau)^{\beta-1}}{\Gamma(\beta)} f(\tau, u(\tau)) d \tau\right) d s \\
& <\operatorname{Ma} \max _{0 \leq t \leq 1} \int_{0}^{1} G(t, s) \phi_{q}\left(\frac{s^{\beta}}{\Gamma(\beta+1)}\right) d s \\
& \leq \operatorname{Ma}_{q}\left(\frac{1}{\Gamma(\beta+1)}\right) \int_{0}^{1} G(s, s) d s=a,
\end{aligned}
$$

which shows that $T: \bar{P}_{a} \rightarrow P_{a}$, that is, $\|T u\|<a$ for $u \in \bar{P}_{a}$. This shows that condition $\left(\mathrm{B}_{2}\right)$ of Lemma 2.7 is satisfied. Finally, we show that $\left(\mathrm{B}_{3}\right)$ of Lemma 2.7 also holds. Assume that $u \in \bar{P}_{\frac{b}{\lambda^{2}}}$ with $\|T u\|>\frac{b}{\lambda^{2}}$, then by the definition of cone $P$, we have

$$
\theta(T u)=\min _{\frac{1}{4} \leq t \leq \frac{3}{4}} T u(t) \geq \lambda\|T u\|>\lambda^{2}\|T u\|=b / \frac{b}{\lambda^{2}}\|T u\| .
$$

So condition $\left(\mathrm{B}_{3}\right)$ of Lemma 2.7 is satisfied. Thus using Lemma 2.7, $T$ has at least two fixed points. Consequently, the boundary value problem (1.1) has at least two positive solutions $u_{1}$ and $u_{2}$ in $\bar{P}_{\frac{b}{\lambda^{2}}}$ satisfying $\left\|u_{1}\right\|<a, \min _{\frac{1}{4} \leq t \leq \frac{3}{4}} u_{2}(t)<b$ and $\left\|u_{2}\right\|>a$. The proof is completed.

Theorem 3.2 Let $f(t, u)$ be nonnegative continuous on $[0,1] \times[0,+\infty)$. Assume that there exist constants $a, b, c$ with $\lambda^{2} c>b>a>0$ such that

$\left(\mathrm{H}_{3}\right) f(t, u)<\phi_{p}(M a)$, for $(t, u) \in[0,1] \times[0, a]$;

$\left(\mathrm{H}_{4}\right) f(t, u) \geq \phi_{p}(N b)$, for $(t, u) \in\left[\frac{1}{4}, \frac{3}{4}\right] \times\left[b, \frac{b}{\lambda^{2}}\right]$;

$\left(\mathrm{H}_{5}\right) f(t, u) \leq \phi_{p}(M c)$, for $(t, u) \in[0,1] \times[0, c]$.

Then the boundary value problem (1.1) has at least three positive solutions $u_{1}, u_{2}$ and $u_{3}$ with $\left\|u_{1}\right\|<a, \min _{\frac{1}{4} \leq t \leq \frac{3}{4}} u_{2}(t)>b,\left\|u_{3}\right\|>a$ and $\min _{\frac{1}{4} \leq t \leq \frac{3}{4}} u_{3}(t)<b$, where $\lambda$ is given as in Lemma 2.5 .

Proof If $u \in \bar{P}_{c}$, then $\|u\| \leq c$. By assumption $\left(\mathrm{H}_{5}\right)$, we have

$$
\begin{aligned}
\|T u\| & =\max _{0 \leq t \leq 1}|T u(t)|=\max _{0 \leq t \leq 1} \int_{0}^{1} G(t, s) \phi_{q}\left(\int_{0}^{s} \frac{(s-\tau)^{\beta-1}}{\Gamma(\beta)} f(\tau, u(\tau)) d \tau\right) d s \\
& \leq M c \max _{0 \leq t \leq 1} \int_{0}^{1} G(t, s) \phi_{q}\left(\frac{s^{\beta}}{\Gamma(\beta+1)}\right) d s \\
& \leq M c \phi_{q}\left(\frac{1}{\Gamma(\beta+1)}\right) \int_{0}^{1} G(s, s) d s=c .
\end{aligned}
$$

This shows that $T: \bar{P}_{c} \rightarrow \bar{P}_{c}$. Using the same arguments as in the proof of Lemma 3.1, we can show that $T: \bar{P}_{c} \rightarrow \bar{P}_{c}$ is a completely continuous operator. It follows from the 
conditions $\left(\mathrm{H}_{3}\right)$ and $\left(\mathrm{H}_{4}\right)$ in Theorem 3.2 that $c>\frac{b}{\lambda^{2}}>b>a$. Similarly with the proof of Theorem 3.1, we have $T: \bar{P}_{a} \rightarrow P_{a}$ and

$$
\left\{u \in P\left(\theta, b, \frac{b}{\lambda^{2}}\right) \mid \theta(u)>b\right\} \neq \emptyset, \quad \theta(T u)>b, \quad \text { for all } u \in P\left(\theta, b, \frac{b}{\lambda^{2}}\right) .
$$

Moreover, for $u \in P(\theta, b, c)$ and $\|T u\|>\frac{b}{\lambda^{2}}$, we have

$$
\theta(T u)=\min _{\frac{1}{4} \leq t \leq \frac{3}{4}} T u(t) \geq \lambda\|T u\|>\frac{b}{\lambda}>b .
$$

So all the conditions of Lemma 2.6 are satisfied. Thus using Lemma 2.6, $T$ has at least three fixed points. So, the boundary value problem (1.1) has at least three positive solutions $u_{1}$, $u_{2}$ and $u_{3}$ with $\left\|u_{1}\right\|<a, \min _{\frac{1}{4} \leq t \leq \frac{3}{4}} u_{2}(t)>b,\left\|u_{3}\right\|>a$ and $\min _{\frac{1}{4} \leq t \leq \frac{3}{4}} u_{3}(t)<b$. The proof is completed.

Theorem 3.3 Let $f(t, u)$ be nonnegative continuous on $[0,1] \times[0,+\infty)$. If the following assumptions are satisfied:

$\left(\mathrm{H}_{6}\right) f_{0}=f_{\infty}=+\infty$;

$\left(\mathrm{H}_{7}\right)$ there exists a constant $\mu_{1}>0$ such that

$$
f(t, u)<\phi_{p}\left(M \mu_{1}\right), \quad \text { for }(t, u) \in[0,1] \times\left[0, \mu_{1}\right],
$$

then the boundary value problem (1.1) has at least two positive solutions $u_{1}$ and $u_{2}$ such that $0<\left\|u_{1}\right\|<\mu_{1}<\left\|u_{2}\right\|$.

Proof From Lemma 3.1, we obtain $T: P \rightarrow P$ is completely continuous. In view of $f_{0}=+\infty$, there exists $\sigma_{1} \in\left(0, \mu_{1}\right)$ such that

$$
f(t, u) \geq \phi_{p}\left(\gamma_{1} u\right), \quad \text { for } 0 \leq t \leq 1,0<u \leq \sigma_{1},
$$

where $\gamma_{1} \in\left(\frac{N}{2},+\infty\right)$.

Let $\Omega_{\sigma_{1}}=\left\{u \in P \mid\|u\|<\sigma_{1}\right\}$. Then, for any $u \in \partial \Omega_{\sigma_{1}}$, we have

$$
\begin{aligned}
T u\left(\frac{1}{2}\right) & =\int_{0}^{1} G\left(\frac{1}{2}, s\right) \phi_{q}\left(\int_{0}^{s} \frac{(s-\tau)^{\beta-1}}{\Gamma(\beta)} f(\tau, u(\tau)) d \tau\right) d s \\
& \geq \int_{\frac{1}{4}}^{\frac{3}{4}} G\left(\frac{1}{2}, s\right) \phi_{q}\left(\int_{0}^{s} \frac{(s-\tau)^{\beta-1}}{\Gamma(\beta)} f(\tau, u(\tau)) d \tau\right) d s \\
& \geq \int_{\frac{1}{4}}^{\frac{3}{4}} G\left(\frac{1}{2}, s\right) \phi_{q}\left(\int_{0}^{s} \frac{(s-\tau)^{\beta-1}}{\Gamma(\beta)} \phi_{p}\left(\gamma_{1} u\right) d \tau\right) d s \\
& \geq \int_{\frac{1}{4}}^{\frac{3}{4}} G\left(\frac{1}{2}, s\right) \phi_{q}\left(\int_{0}^{s} \frac{(s-\tau)^{\beta-1}}{\Gamma(\beta)} \phi_{p}\left(\gamma_{1} \lambda\|u\|\right) d \tau\right) d s \\
& \geq \gamma_{1} \lambda\|u\| \phi_{q}\left(\frac{1}{4^{\beta} \Gamma(\beta+1)}\right) \int_{\frac{1}{4}}^{\frac{3}{4}} G\left(\frac{1}{2}, s\right) d s \\
& =\gamma_{1} \lambda\|u\| \frac{2}{\lambda N}=\frac{2 \gamma_{1}}{N}\|u\|>\|u\|,
\end{aligned}
$$


which implies $\|T u\|>\|u\|$ for $u \in \partial \Omega_{\sigma_{1}}$. Hence, Lemma 2.9 implies

$$
i\left(T, \Omega_{\sigma_{1}}, P\right)=0 .
$$

On the other hand, since $f_{\infty}=+\infty$, there exists $\sigma_{3}>\mu_{1}$ such that

$$
f(t, u) \geq \phi_{p}\left(\gamma_{2} u\right), \quad \text { for } u \geq \sigma_{3}
$$

where $\gamma_{2} \in\left(\frac{N}{2},+\infty\right)$.

Let $\sigma_{2}>\max \left\{\frac{\sigma_{3}}{\lambda}, \mu_{1}\right\}$ and $\Omega_{\sigma_{2}}=\left\{u \in P \mid\|u\|<\sigma_{2}\right\}$. Then $\min _{\frac{1}{4} \leq t \leq \frac{3}{4}} u(t) \geq \lambda\|u\|>\sigma_{3}$, for any $u \in \partial \Omega_{\sigma_{2}}$. By using the method to get (3.1), we obtain

$$
T u\left(\frac{1}{2}\right)>\frac{2 \gamma_{2}}{N}\|u\|>\|u\|
$$

which implies $\|T u\|>\|u\|$ for $u \in \partial \Omega_{\sigma_{2}}$. Thus, from Lemma 2.9, we have

$$
i\left(T, \Omega_{\sigma_{2}}, P\right)=0 .
$$

Finally, let $\Omega_{\mu_{1}}=\left\{u \in P \mid\|u\|<\mu_{1}\right\}$. Then, for any $u \in \partial \Omega_{\mu_{1}}$, by $\left(\mathrm{H}_{7}\right)$, we then get

$$
\begin{aligned}
T u(t) & =\int_{0}^{1} G(t, s) \phi_{q}\left(\int_{0}^{s} \frac{(s-\tau)^{\beta-1}}{\Gamma(\beta)} f(\tau, u(\tau)) d \tau\right) d s \\
& \leq \int_{0}^{1} G(s, s) \phi_{q}\left(\int_{0}^{s} \frac{(s-\tau)^{\beta-1}}{\Gamma(\beta)} f(\tau, u(\tau)) d \tau\right) d s \\
& <\int_{0}^{1} G(s, s) \phi_{q}\left(\int_{0}^{s} \frac{(s-\tau)^{\beta-1}}{\Gamma(\beta)} \phi_{p}\left(M \mu_{1}\right) d \tau\right) d s \\
& \leq M \mu_{1} \phi_{q}\left(\frac{1}{\Gamma(\beta+1)}\right) \int_{0}^{1} G(s, s) d s=\mu_{1}=\|u\|,
\end{aligned}
$$

which implies $\|T u\|<\|u\|$ for $u \in \partial \Omega_{\mu_{1}}$. Using Lemma 2.9 again, we get

$$
i\left(T, \Omega_{\mu_{1}}, P\right)=1 .
$$

Note that $\sigma_{1}<\mu_{1}<\sigma_{2}$, by the additivity of fixed-point index and (3.1)-(3.3), we obtain

$$
i\left(T, \Omega_{\mu_{1}} \backslash \bar{\Omega}_{\sigma_{1}}, P\right)=i\left(T, \Omega_{\mu_{1}}, P\right)-i\left(T, \Omega_{\sigma_{1}}, P\right)=1
$$

and

$$
i\left(T, \Omega_{\sigma_{2}} \backslash \bar{\Omega}_{\mu_{1}}, P\right)=i\left(T, \Omega_{\sigma_{2}}, P\right)-i\left(T, \Omega_{\mu_{1}}, P\right)=-1 .
$$

Hence, $T$ has a fixed point $u_{1}$ in $\Omega_{\mu_{1}} \backslash \bar{\Omega}_{\sigma_{1}}$, and has a fixed point $u_{2}$ in $\Omega_{\sigma_{2}} \backslash \bar{\Omega}_{\mu_{1}}$. Clearly, $u_{1}$ and $u_{2}$ are positive solutions of the boundary value problem (1.1) and $0<\left\|u_{1}\right\|<\mu_{1}<\left\|u_{2}\right\|$. The proof is completed. 
Theorem 3.4 Let $f(t, u)$ be nonnegative continuous on $[0,1] \times[0,+\infty)$. If the following assumptions are satisfied:

$\left(\mathrm{H}_{8}\right) f_{0}=f_{\infty}=0$;

$\left(\mathrm{H}_{9}\right)$ there exists a constant $\mu_{2}>0$ such that

$$
f(t, u)>\phi_{p}\left(N \mu_{2}\right), \quad \text { for }(t, u) \in\left[\frac{1}{4}, \frac{3}{4}\right] \times\left[\lambda \mu_{2}, \mu_{2}\right] .
$$

Then the boundary value problem (1.1) has at least two positive solutions $u_{1}$ and $u_{2}$ such that $0<\left\|u_{1}\right\|<\mu_{2}<\left\|u_{2}\right\|$.

Proof From Lemma 3.1, we obtain $T: P \rightarrow P$ is completely continuous. In view of $f_{0}=0$, there exists $\delta_{1} \in\left(0, \mu_{2}\right)$ such that

$$
f(t, u) \leq \phi_{p}\left(\kappa_{1} u\right), \quad \text { for } 0 \leq t \leq 1,0<u \leq \delta_{1},
$$

where $\kappa_{1} \in(0, M)$.

Let $\Omega_{\delta_{1}}=\left\{u \in P \mid\|u\|<\delta_{1}\right\}$. Then, for any $u \in \partial \Omega_{\delta_{1}}$, we have

$$
\begin{aligned}
T u(t) & \leq \int_{0}^{1} G(s, s) \phi_{q}\left(\int_{0}^{s} \frac{(s-\tau)^{\beta-1}}{\Gamma(\beta)} f(\tau, u(\tau)) d \tau\right) d s \\
& \leq \int_{0}^{1} G(s, s) \phi_{q}\left(\int_{0}^{s} \frac{(s-\tau)^{\beta-1}}{\Gamma(\beta)} \phi_{p}\left(\kappa_{1} u\right) d \tau\right) d s \\
& \leq \int_{0}^{1} G(s, s) \phi_{q}\left(\int_{0}^{s} \frac{(s-\tau)^{\beta-1}}{\Gamma(\beta)} \phi_{p}\left(\kappa_{1}\|u\|\right) d \tau\right) d s \\
& \leq \kappa_{1}\|u\| \phi_{q}\left(\frac{1}{\Gamma(\beta+1)}\right) \int_{0}^{1} G(s, s) d s=\frac{\kappa_{1}}{M}\|u\|<\|u\|,
\end{aligned}
$$

which implies $\|T u\|<\|u\|$ for $u \in \partial \Omega_{\delta_{1}}$. Hence, Lemma 2.9 implies

$$
i\left(T, \Omega_{\delta_{1}}, P\right)=1 .
$$

Next, since $f_{\infty}=0$, there exists $\delta_{3}>\mu_{2}$ such that

$$
f(t, u) \leq \phi_{p}\left(\kappa_{2} u\right), \quad \text { for } u \geq \delta_{3},
$$

where $\kappa_{2} \in(0, M)$. We consider two cases.

Case 1: Suppose that $f$ is bounded, which implies that there exists $M^{*}>0$ such that $f(t, u) \leq \phi_{p}\left(M^{*}\right)$ for all $t \in[0,1]$ and $u \in[0,+\infty)$.

Take $\delta_{4}>\max \left\{\frac{M^{*}}{M}, \delta_{3}\right\}$. Then, for $u \in P$ with $\|u\|=\delta_{4}$, we get

$$
\begin{aligned}
\operatorname{Tu}(t) & \leq \int_{0}^{1} G(s, s) \phi_{q}\left(\int_{0}^{s} \frac{(s-\tau)^{\beta-1}}{\Gamma(\beta)} f(\tau, u(\tau)) d \tau\right) d s \\
& \leq \int_{0}^{1} G(s, s) \phi_{q}\left(\int_{0}^{s} \frac{(s-\tau)^{\beta-1}}{\Gamma(\beta)} \phi_{p}\left(M^{*}\right) d \tau\right) d s \\
& \leq M^{*} \phi_{q}\left(\frac{1}{\Gamma(\beta+1)}\right) \int_{0}^{1} G(s, s) d s=\frac{M^{*}}{M}<\delta_{4}=\|u\| .
\end{aligned}
$$


Case 2: Suppose that $f$ is unbounded. In view of $f:[0,1] \times[0,+\infty) \rightarrow[0,+\infty)$ being continuous, there exist $t^{*} \in[0,1]$ and $\delta_{5}>\max \left\{\frac{\delta_{3}}{\lambda}, \mu_{2}\right\}$ such that

$$
f(t, u) \leq f\left(t^{*}, \delta_{5}\right), \quad \text { for } 0 \leq t \leq 1,0 \leq u \leq \delta_{5} .
$$

Then, for $u \in P$ with $\|u\|=\delta_{5}$, we obtain

$$
\begin{aligned}
T u(t) & \leq \int_{0}^{1} G(s, s) \phi_{q}\left(\int_{0}^{s} \frac{(s-\tau)^{\beta-1}}{\Gamma(\beta)} f(\tau, u(\tau)) d \tau\right) d s \\
& \leq \int_{0}^{1} G(s, s) \phi_{q}\left(\int_{0}^{s} \frac{(s-\tau)^{\beta-1}}{\Gamma(\beta)} f\left(t^{*}, \delta_{5}\right) d \tau\right) d s \\
& \leq \int_{0}^{1} G(s, s) \phi_{q}\left(\int_{0}^{s} \frac{(s-\tau)^{\beta-1}}{\Gamma(\beta)} \phi_{p}\left(\kappa_{2}\|u\|\right) d \tau\right) d s \\
& \leq \kappa_{2} \delta_{5} \phi_{q}\left(\frac{1}{\Gamma(\beta+1)}\right) \int_{0}^{1} G(s, s) d s=\frac{\kappa_{2} \delta_{5}}{M}<\delta_{5}=\|u\| .
\end{aligned}
$$

So, in either case, if we always choose $\Omega_{\delta_{2}}=\left\{u \in E \mid\|u\|<\delta_{2}=\max \left\{\delta_{4}, \delta_{5}\right\}\right\}$, then we have

$$
\|T u\|<\|u\|, \quad \text { for } u \in \partial \Omega_{\delta_{2}} .
$$

Thus, from Lemma 2.9, we have

$$
i\left(T, \Omega_{\delta_{2}}, P\right)=1 .
$$

Finally, Let $\Omega_{\mu_{2}}=\left\{u \in P \mid\|u\|<\mu_{2}\right\}$. Then, for any $u \in \partial \Omega_{\mu_{2}}, \min _{\frac{1}{4} \leq t \leq \frac{3}{4}} u(t) \geq \lambda\|u\|=$ $\lambda \mu_{2}$, by $\left(\mathrm{H}_{9}\right)$, and we then obtain

$$
\begin{aligned}
T u\left(\frac{1}{2}\right) & =\int_{0}^{1} G\left(\frac{1}{2}, s\right) \phi_{q}\left(\int_{0}^{s} \frac{(s-\tau)^{\beta-1}}{\Gamma(\beta)} f(\tau, u(\tau)) d \tau\right) d s \\
& \geq \int_{\frac{1}{4}}^{\frac{3}{4}} G\left(\frac{1}{2}, s\right) \phi_{q}\left(\int_{0}^{s} \frac{(s-\tau)^{\beta-1}}{\Gamma(\beta)} f(\tau, u(\tau)) d \tau\right) d s \\
& \geq \int_{\frac{1}{4}}^{\frac{3}{4}} G\left(\frac{1}{2}, s\right) \phi_{q}\left(\int_{0}^{s} \frac{(s-\tau)^{\beta-1}}{\Gamma(\beta)} \phi_{p}\left(N \mu_{2}\right) d \tau\right) d s \\
& \geq N \mu_{2} \phi_{q}\left(\frac{1}{4^{\beta} \Gamma(\beta+1)}\right) \int_{\frac{1}{4}}^{\frac{3}{4}} G\left(\frac{1}{2}, s\right) d s=\frac{2 \mu_{2}}{\lambda}>\mu_{2}=\|u\|,
\end{aligned}
$$

which implies $\|T u\|>\|u\|$ for $u \in \partial \Omega_{\mu_{2}}$. An application of Lemma 2.9 again shows that

$$
i\left(T, \Omega_{\mu_{2}}, P\right)=0 .
$$

Note that $\delta_{1}<\mu_{2}<\delta_{2}$; by the additivity of fixed-point index and (3.4)-(3.6), we obtain

$$
i\left(T, \Omega_{\mu_{2}} \backslash \bar{\Omega}_{\delta_{1}}, P\right)=i\left(T, \Omega_{\mu_{2}}, P\right)-i\left(T, \Omega_{\delta_{1}}, P\right)=-1
$$

and

$$
i\left(T, \Omega_{\delta_{2}} \backslash \bar{\Omega}_{\mu_{2}}, P\right)=i\left(T, \Omega_{\delta_{2}}, P\right)-i\left(T, \Omega_{\mu_{2}}, P\right)=1 .
$$


Hence, $T$ has a fixed point $u_{1}$ in $\Omega_{\mu_{2}} \backslash \bar{\Omega}_{\delta_{1}}$, and it has a fixed point $u_{2}$ in $\Omega_{\delta_{2}} \backslash \bar{\Omega}_{\mu_{2}}$. Consequently, $u_{1}$ and $u_{2}$ are positive solutions of the boundary value problem (1.1) and $0<$ $\left\|u_{1}\right\|<\mu_{2}<\left\|u_{2}\right\|$. The proof is completed.

\section{Example}

In this section, we present an example to illustrate the main result.

Example 4.1 We consider the boundary value problem of the fractional differential equation

$$
\left\{\begin{array}{l}
D_{0^{+}}^{\frac{1}{2}}\left(\phi_{p}\left(D_{0^{+}}^{\frac{3}{2}} u(t)\right)\right)+f(t, u(t))=0, \quad 0<t<1, \\
\frac{1}{2} u(0)-u^{\prime}(0)=0, \quad \frac{1}{2} u(1)+\frac{1}{2} u^{\prime}(1)=0, \quad D_{0^{+}}^{\alpha} u(0)=0,
\end{array}\right.
$$

where

$$
f(t, u)= \begin{cases}\frac{t}{100}+22 u^{2}, & \text { for } u \leq 2 \\ \frac{t}{100}+u+86, & \text { for } u>2\end{cases}
$$

Let $p=2$. We note that $\alpha=\frac{3}{2}, \beta=\frac{1}{2}, \xi=\gamma=\delta=\frac{1}{2}, \eta=1$. By a simple calculation, we obtain $\rho=1, \lambda=\frac{1}{5}<1$ and

$$
\begin{aligned}
& M=\phi_{q}(\Gamma(\beta+1))\left(\int_{0}^{1} G(s, s) d s\right)^{-1}=1.3287 \\
& N=\frac{2}{\lambda} \phi_{q}\left(4^{\beta} \Gamma(\beta+1)\right)\left(\int_{\frac{1}{4}}^{\frac{3}{4}} G\left(\frac{1}{2}, s\right) d s\right)^{-1}=43.1569 .
\end{aligned}
$$

Choosing $a=0.01, b=2, c=262$, evidently, $\lambda^{2} c>b>a>0$ and

$$
\begin{aligned}
& f(t, u)=\frac{t}{100}+22 u^{2} \leq 0.0122<\phi_{2}(M a)=0.013287, \quad \text { for }(t, u) \in[0,1] \times[0,0.01] \\
& f(t, u)=\frac{t}{100}+u+86 \geq 88.0025>\phi_{2}(N b) \approx 86.3138, \quad \text { for }(t, u) \in\left[\frac{1}{4}, \frac{3}{4}\right] \times[2,50] \\
& f(t, u)=\frac{t}{100}+u+86 \leq 348.01<\phi_{2}(M c)=348.1194, \quad \text { for }(t, u) \in[0,1] \times[0,262]
\end{aligned}
$$

Consequently, all the conditions of Theorem 3.2 are satisfied. With the use of Theorem 3.2, the boundary value problem (4.1) has at least three positive solutions $u_{1}, u_{2}$, and $u_{3}$ with

$$
\left\|u_{1}\right\|<0.01, \quad \min _{\frac{1}{4} \leq t \leq \frac{3}{4}} u_{2}(t)>2, \quad\left\|u_{3}\right\|>0.01, \quad \text { and } \quad \min _{\frac{1}{4} \leq t \leq \frac{3}{4}} u_{3}(t)<2 .
$$

\section{Conclusion}

In this paper, the Sturm-Liouville boundary value problems of fractional differential equations with $p$-Laplacian are investigated, the existence of at least two or at least three positive solutions for the fractional differential equations with Robin boundary conditions are given by using Leggett-Williams fixed-point theorems and the fixed-point index theory, respectively. 
It is worth emphasizing that our work presented in this article has the following features: Firstly, the boundary conditions in (1.1) are important Robin boundary conditions. Secondly, our results improve and extend the main results of $[11,12]$ for the Sturm-Liouville boundary value problems of integer-order differential equations with $p$-Laplacian. For example, if $\alpha=2, \beta=1$, then the problem (1.1) reduces to

$$
\left\{\begin{array}{l}
\left(\phi_{p}\left(u^{\prime \prime}(t)\right)^{\prime}+f(t, u(t))=0, \quad 0<t<1,\right. \\
\xi u(0)-\eta u^{\prime}(0)=0, \quad \gamma u(1)+\delta u^{\prime}(1)=0, \quad u^{\prime \prime}(0)=0,
\end{array}\right.
$$

which is studied in $[11,12]$. Furthermore, if we take $p=2$, problem (1.1) is the usual form of third-order Sturm-Liouville boundary value problem

$$
\begin{cases}\left.u^{\prime \prime \prime}(t)\right)+f(t, u(t))=0, & 0<t<1, \\ \xi u(0)-\eta u^{\prime}(0)=0, & \gamma u(1)+\delta u^{\prime}(1)=0, \quad u^{\prime \prime}(0)=0 .\end{cases}
$$

The method can be applied on the Sturm-Liouville boundary value problems of higherorder fractional differential equations with $p$-Laplacian and boundary conditions involving fractional derivatives

$$
\left\{\begin{array}{l}
D_{0^{+}}^{\beta}\left(\phi_{p}\left(D_{0^{+}}^{\alpha} u(t)\right)\right)+f(t, u(t))=0, \quad 0<t<1, \\
\xi u(0)-\eta D^{\gamma} u(0)=0, \quad \gamma u(1)+\delta D^{\gamma} u(0)=0, \quad D_{0^{+}}^{\alpha} u(0)=0 .
\end{array}\right.
$$

Based on this paper, one can consider boundary value problems of fractional differential equations with parameters, and also one can do further research on eigenvalue problems of fractional differential equations with $p$-Laplacian.

Competing interests

The authors declare that they have no competing interests.

\section{Authors' contributions}

The authors declare that the study was realized in collaboration with the same responsibility. All authors read and approved the final manuscript.

\section{Acknowledgements \\ The authors sincerely thank the reviewers for their valuable suggestions and useful comments that have led to the present improved version of the original manuscript. This research is supported by the Natural Science Foundation of China (11071143, 61374074), Natural Science Outstanding Youth Foundation of Shandong Province (JQ201119) and supported by Shandong Provincial Natural Science Foundation (ZR2012AM009).}

Received: 17 August 2013 Accepted: 10 January 2014 Published: 30 Jan 2014

\section{References}

1. Diaz, Jl, De Thelin, F: On a nonlinear parabolic problem arising in some models related to turbulent flows. SIAM J. Math. Anal. 25(4), 1085-1111 (1994)

2. Evans, LC, Gangbo, W: Differential equations methods for the Monge-Kantorovich mass transfer problem. Mem. Am. Math. Soc. 137(653), 1-75 (1999)

3. Oruganti, S, Shi, J, Shivaji, R: Logistic equation with $p$-Laplacian and constant yield harvesting. Abstr. Appl. Anal. 2004(9), 723-727 (2004)

4. Ly, I, Seck, D: Isoperimetric inequality for an interior free boundary problem with $p$-Laplacian operator. Electron. J. Differ. Equ. 2004(109), 1-12 (2004)

5. Ramaswamy, M, Shivaji, R: Multiple positive solutions for classes of $p$-Laplacian equations. Differ. Integral Equ. 17(11-12), 1255-1261 (2004)

6. Wang, Y, Hou, C: Existence of multiple positive solutions for one-dimensional p-Laplacian. J. Math. Anal. Appl. 315(1), 144-153 (2006)

7. Lian, $\mathrm{H}, \mathrm{Ge}, \mathrm{W}$ : Positive solutions for a four-point boundary value problem with the $p$-Laplacian. Nonlinear Anal. 68(11), 3493-3503 (2008) 
8. Sun, $B, Q u, Y, G e, W$ : Existence and iteration of positive solutions for a multipoint one-dimensional p-Laplacian boundary value problem. Appl. Math. Comput. 197(1), 389-398 (2008)

9. Zhao, X, Ge, W: Successive iteration and positive symmetric solution for a Sturm-Liouville-like four-point boundary value problem with a $p$-Laplacian operator. Nonlinear Anal. 71(11), 5531-5544 (2009)

10. Hai, DD: On singular Sturm-Liouville boundary-value problems. Proc. R. Soc. Lond., Ser. A, Math. Phys. Eng. Sci. 140(1), 49-63 (2010)

11. Zhai, C, Guo, C: Positive solutions for third-order Sturm-Liouville boundary-value problems with $p$-Laplacian. Electron. J. Differ. Equ. 2009(154), 1-9 (2009)

12. Yang, C, Yan, J: Positive solutions for third-order Sturm-Liouville boundary value problems with $p$-Laplacian. Comput. Math. Appl. 59(6), 2059-2066 (2010)

13. Oldham, KB, Spanier, J: The Fractional Calculus. Academic Press, New York (1974)

14. Podlubny, I: Fractional Differential Equations, Mathematics in Science and Engineering. Academic Press, New York (1999)

15. Meral, FC, Royston, TJ, Magin, R: Fractional calculus in viscoelasticity: an experimental study. Commun. Nonlinear Sci. Numer. Simul. 15(4), 939-945 (2010)

16. Tenreiro Machado, J, Kiryakova, V. Mainardi, F: Recent history of fractional calculus. Commun. Nonlinear Sci. Numer. Simul. 16(3), 1140-1153 (2011)

17. Kilbas, AA, Srivastava, HM, Trujillo, JJ: Theory and Applications of Fractional Differential Equations. Elsevier, Amsterdam (2006)

18. Zhao, Y, Sun, S, Han, Z, Zhang, M: Positive solutions for boundary value problems of nonlinear fractional differential equations. Appl. Math. Comput. 217(16), 6950-6958 (2011)

19. Feng, W, Sun, S, Han, Z, Zhao, Y: Existence of solutions for a singular system of nonlinear fractional differential equations. Comput. Math. Appl. 62(3), 1370-1378 (2011)

20. Zhao, Y, Sun, S, Han, Z, Li, Q: The existence of multiple positive solutions for boundary value problems of nonlinear fractional differential equations. Commun. Nonlinear Sci. Numer. Simul. 16(4), 2086-2097 (2011)

21. Nyamoradi, N, Bashiri, T: Multiple positive solutions for nonlinear fractional differential systems. Fract. Differ. Calc. 2(2) 119-128 (2012)

22. Ahmad, B, Nieto, JJ, Alsaedi, A, El-Shahed, M: A study of nonlinear Langevin equation involving two fractional orders in different intervals. Nonlinear Anal., Real World Appl. 13(2), 599-606 (2012)

23. Han, Z, Lu, H, Sun, S, Yang, D: Positive solutions to boundary value problems of $p$-Laplacian fractional differential equations with a parameter in the boundary conditions. Electron. J. Differ. Equ. 2012(213), 1-14 (2012)

24. Chen, T, Liu, W: An anti-periodic boundary value problem for fractional differential equation with $p$-Laplacian operator. Appl. Math. Lett. 25(11), 1671-1675 (2012)

25. Chai, G: Positive solutions for boundary value problem of fractional differential equation with $p$-Laplacian operator. Bound. Value Probl. 2012, 1-18 (2012)

26. Wang, J, Xiang, H: Upper and lower solutions method for a class of singular fractional boundary value problems with p-Laplacian operator. Abstr. Appl. Anal. 2010, Article ID 971824 (2010). doi:10.1155/2010/971824

27. Dix, JG, Karakostas, GL: A fixed-point theorem for S-type operators on Banach spaces and its applications to boundary-value problems. Nonlinear Anal. 71(9), 3872-3880 (2009)

28. Xu, J, Wei, Z, Dong, W: Uniqueness of positive solutions for a class of fractional boundary value problems. Appl. Math Lett. 25(3), 590-593 (2012)

29. Chen, T, Liu, W, Hu, Z: A boundary value problem for fractional differential equation with $p$-Laplacian operator at resonance. Nonlinear Anal. 75(6), 3210-3217 (2012)

30. Zhao, $Y, C$ Chen, $H$, Huang, L: Existence of positive solutions for nonlinear fractional functional differential equation. Comput. Math. Appl. 64(10), 3456-3467 (2012)

31. Guo, D, Lakshmikantham, V: Nonlinear Problems in Abstract Cones. Academic Press, Orlando (1988)

32. Leggett, RW. Williams, LR: Multiple positive fixed points of nonlinear operators on ordered Banach spaces. Indiana Univ. Math. J. 28(4), 673-688 (1979)

33. Deimling, K: Nonlinear Functional Analysis. Springer, New York (1985)

10.1186/1687-2770-2014-26

Cite this article as: Lu et al.: Multiplicity of positive solutions for Sturm-Liouville boundary value problems of fractional differential equations with p-Laplacian. Boundary Value Problems 2014, 2014:26

\section{Submit your manuscript to a SpringerOpen ${ }^{\circ}$ journal and benefit from:}

- Convenient online submission

- Rigorous peer review

- Immediate publication on acceptance

- Open access: articles freely available online

- High visibility within the field

- Retaining the copyright to your article 